\title{
Real-Time Monitoring for Data Greenhouse Based on Raspberry Pi Technology
}

\author{
Ibrahim Elkhier Hussein Arfeen'1, Abd Elrazig Awadelseed Edries Suliman² \\ ${ }^{1}$ Electrical Department, College of Engineering, AL-Baha University, AL-Baha, KSA \\ ${ }^{2}$ Department of Information System, College of Computers \& Information Technology, University Bisha, Bisha, KSA \\ Email: abodiawad@gmail.com
}

How to cite this paper: Arfeen, I.E.H. and Suliman, A.E.A.E. (2019) Real-Time Monitoring for Data Greenhouse Based on Raspberry Pi Technology. Open Access Library Journal, 6: e5138.

https://doi.org/10.4236/oalib.1105138

Received: December 24, 2018

Accepted: March 16, 2019

Published: March 19, 2019

Copyright (๑) 2019 by author(s) and Open Access Library Inc.

This work is licensed under the Creative Commons Attribution International License (CC BY 4.0).

http://creativecommons.org/licenses/by/4.0/

\begin{abstract}
The greenhouse needs to control environmental parameters to obtain the ideal growth of the plant. The scopes of this paper are to design and implement system of real-time remote light intensity, temperature and humidity monitoring system for greenhouse based on raspberry Pi3 platforms, which enable the user to monitor acquisition data from the greenhouse. The design system, implemented using LabVIEW as platform software, has to receive data acquisition from raspberry Pi3 through the wireless network. The practical result that has been obtained by the system is reliable and it has good performance monitoring convenient.
\end{abstract}

\section{Subject Areas}

Applications of Communication Systems

\section{Keywords}

Raspberry Pi, Greenhouse, Monitoring, Wireless Network

\section{Introduction}

The greenhouse industry is the fastest growing sector worldwide. The greenhouse separates the crop from the environment, thus providing some way of shelter from the direct influence of the external weather conditions. This enables the production of crops that otherwise could not be produced at that specific location [1] [2].

The greenhouse is a structure with walls and roof made chiefly of transparent material, such as glass, in which plants requiring regulated climatic conditions are grown. These structures range in size from small sheds to industrial-sized 
buildings. A miniature greenhouse is known as a cold frame. The interior of a greenhouse exposed to sunlight becomes significantly warmer than the external ambient temperature, protecting its contents in cold weather.

Electronic monitoring of areas of environmental interest has recently attracted considerable research interest, at a national and international level, [3] [4]. The increasing concern of developed societies for the environment is reflected in current research and development while, at the same time, recent advances in electronics, computers and communications offer realistic (semi-)automated monitoring solutions, for scientific or decision-making purposes (protection, prevention). The use of Wireless Sensor Networks (WSNs) in precision agriculture increases the efficiency, productivity, and profitability of many agricultural production systems.

The paper is organized in the following sequence: first, the raspberry pi wireless sensor networks using the personal computer through LabVIEW platform for industrial automation and control, and explaining the overall system of the sensor section and a host section; second, the software flow diagrams of the system; thirdly, the experimentation of design system based on wireless communication control system and results. The last section is discussions and conclusions, followed by the references.

\subsection{Raspberry Pi Technology and LabVIEW}

This section gives the information about Raspberry Pi, which is the major component used; different topologies are used to form the network and the comparison of Raspberry Pi, LabVIEW, and other wireless technologies.

\subsubsection{Raspberry Pi3 Board}

The core of the hardware part is raspberry pi which receives a signal from a group of sensors and transmitting data acquisition to PC via interface software. The new Raspberry Pi3 is built on the back of the Broadcom BCM2836. The BCM2836 is a system-on-a-chip processor containing four ARM cores and Broadcom's VideoCore ${ }^{\circledR}$ IV graphics stack. On top of this, several other components make up the device, including USB, RCA, and micro SD card storage. The Raspberry Pi3, a microprocessor that can run on the Linux operating system. Figure 1 illustrates the raspberry Pi3 model B.

\subsubsection{LabVIEW Software}

The software is an integrated package which is developing under the graphical program structure in LabVIEW 2014 for data acquisition from Greenhouse. LabVIEW (Laboratory Virtual Instrument Engineering Workbench) is the graphical programming language. LabVIEW system design software is at the center of the National Instruments platform. Providing complete tools that are needed to build any measurement or control application.

\subsection{LabVIEW Interfacing for Raspberry Pi3}

At its heart, LINX is a hardware abstraction layer that allows you to have a single 


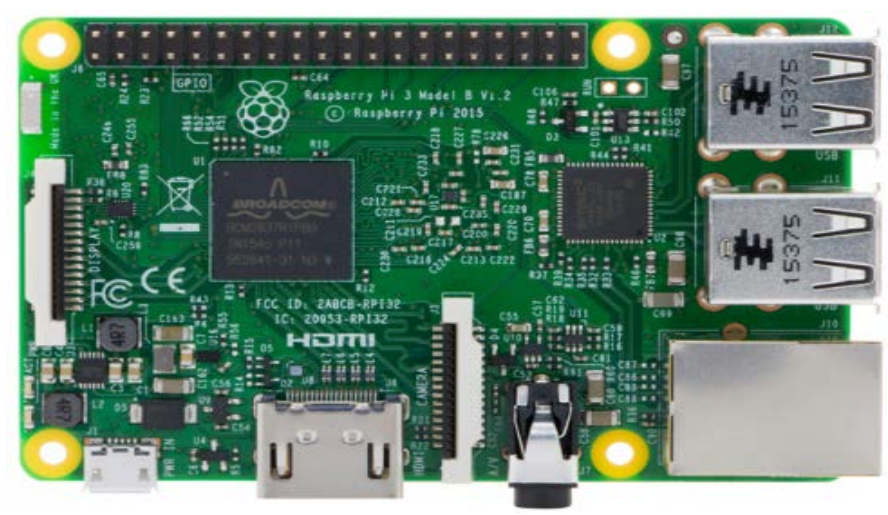

Figure 1. Raspberry Pi3 Model B.

LabVIEW interface to a variety of different hardware devices. Depending on the device, you can access it in one of two ways: Remote or Local I/O (Figure 2).

\section{Embedded System Design}

The main objective of this paper, allows users to monitor data environmental form Greenhouse farming to enhance productivity. The acquired data from greenhouse by sensors, which connected into raspberry pi3 port. Theses acquire data uploaded to a computer via the warless network.

\subsection{System Architecture}

The block diagram of the system shows in Figure 3.

\subsection{Hardware}

This is section include a group of sensors and the main circuit connected to Raspberry Pi3.

\section{Group of Sensors}

The task of this group to convert physical phenomena from greenhouse to electrical signal.

\section{1) Temperature and Humidity Sensor}

DHT11 digital temperature and humidity sensor is a composite Sensor contain a calibrated digital signal output of the temperature and humidity, see Figure 4. This sensor includes a resistive-type humidity measurement component and it is a negative temperature coefficient of resistivity (NTC) temperature measurement component.

The output is directly connected with the Raspberry $\mathrm{Pi} 3 \mathrm{I} / \mathrm{O}$ port pin (7).

\section{2) Light Dependent Resistor (LDR)}

The Photosensitive resistance light sensor is used to adjust the amount of light need for a greenhouse. Figure 5 illustrates the basic resistance light sensor circuit. The most commonly used of all photo-resistive light sensors being Cadmium Sulphide. An LDR is a component that has a resistance that changes with the light intensity that falls upon it. 


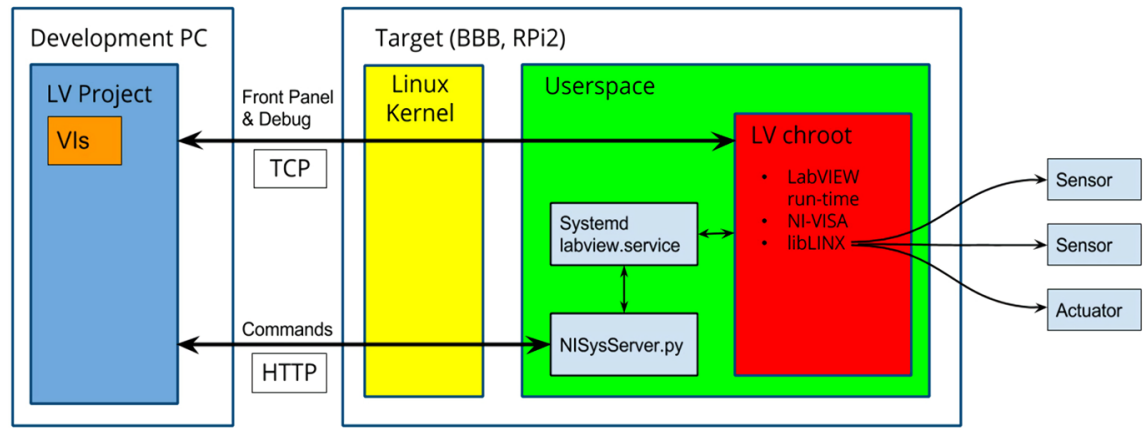

Figure 2. LabVIEW interfacing with raspberry Pi.

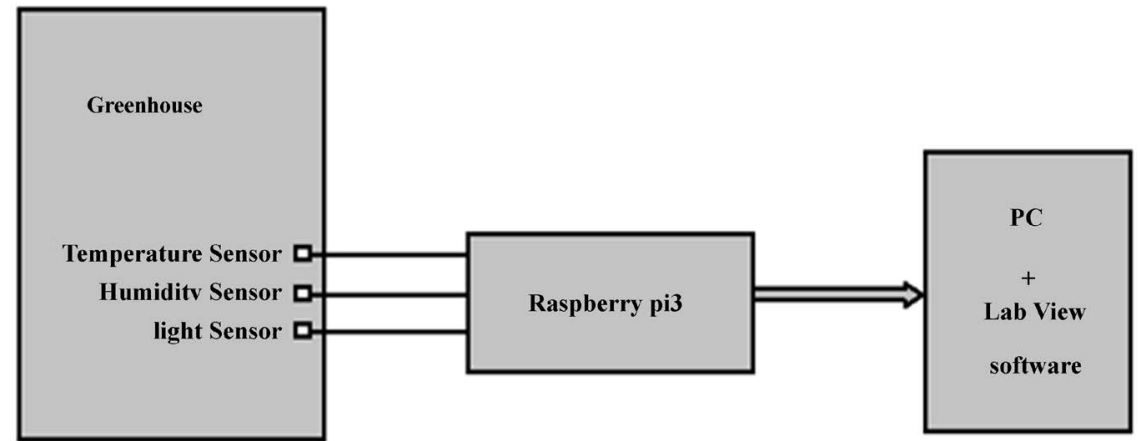

Figure 3. Block diagram for practical design.

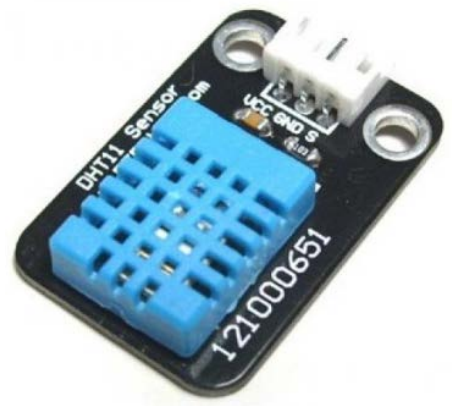

Figure 4. DHT11 temperature and humidity sensors.

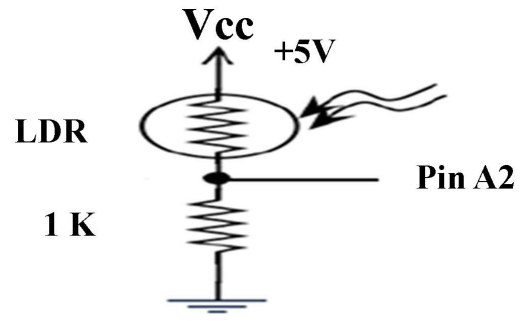

Figure 5. Light sensor circuit.

\section{3) Convert Analog Digital (ADC0804)}

The reason for used ADC0804 to convert the analog voltage from photosensitive resistance light sensor to the digital signal to adapt raspberry pi3 input port (no analog input port on raspberry Pi3). Figure 6 shows the pin diagram for an 
ADC0804 8-bit AID converter IC. The output of ADC0804 connected to the voltage divider to adapt raspberry pi3 inputs voltage $(3.3 \mathrm{~V})$.

\section{4) The main Circuit Design}

Figure 7 and Figure 8 explain a group of sensors circuits to connected to raspberry Pi3.

\subsection{Software}

LabVIEW Interface for Raspberry Pi3 by Maker Hub toolkit it is allows developers to acquire data from raspberry $\mathrm{Pi} 3$ via wireless network and process it in the LabVIEW.

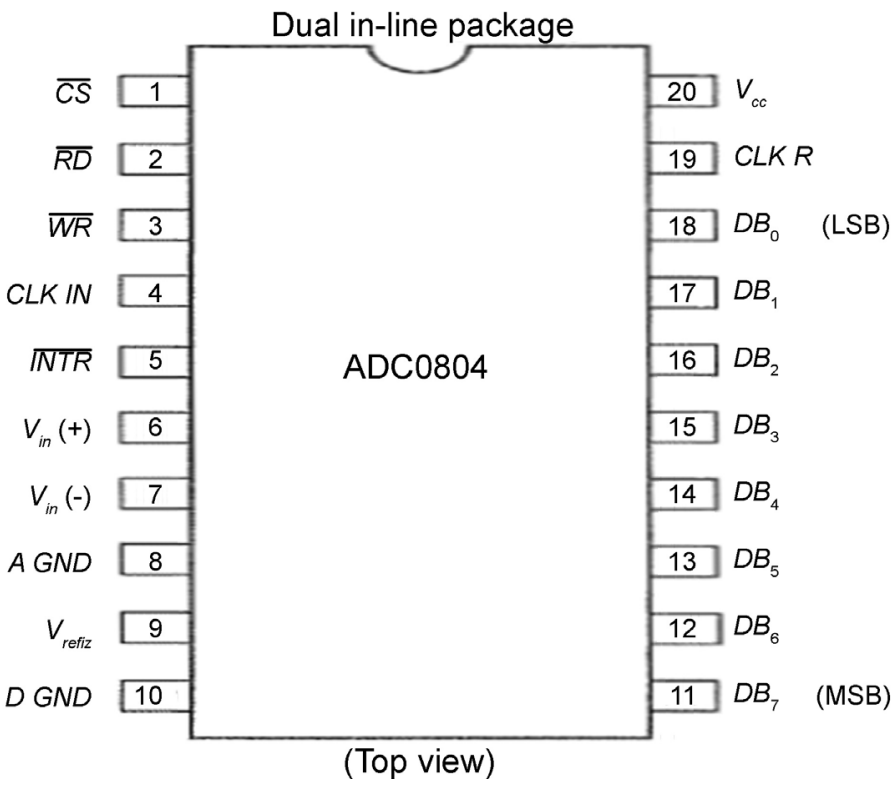

Figure 6. Diagram for an ADC0804.

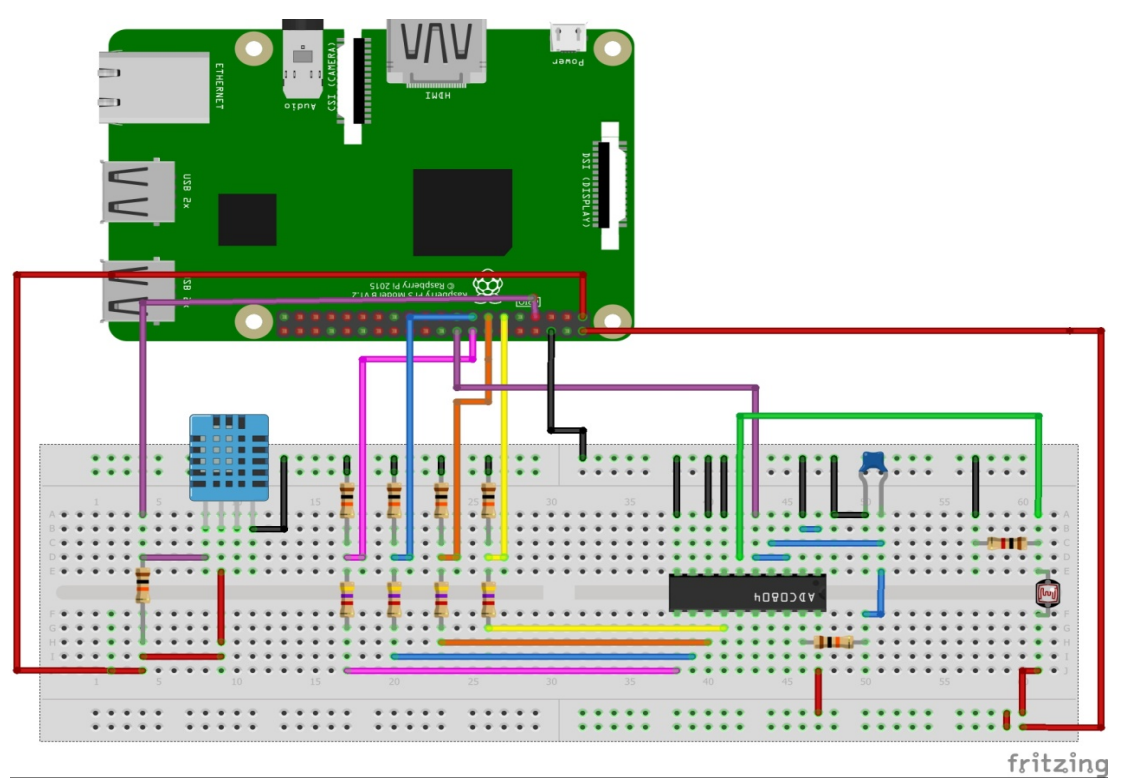

Figure 7. Main circuit design. 


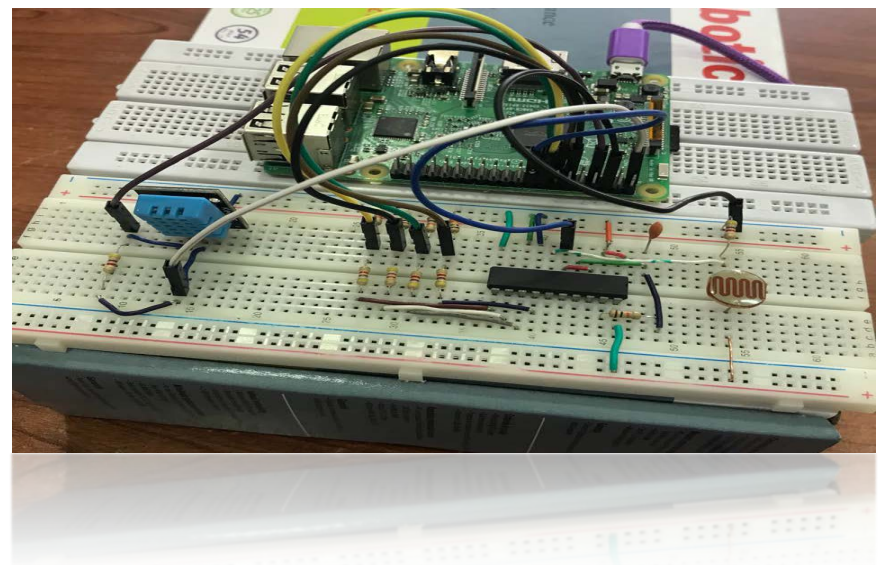

Figure 8. Main circuit design physical implement.

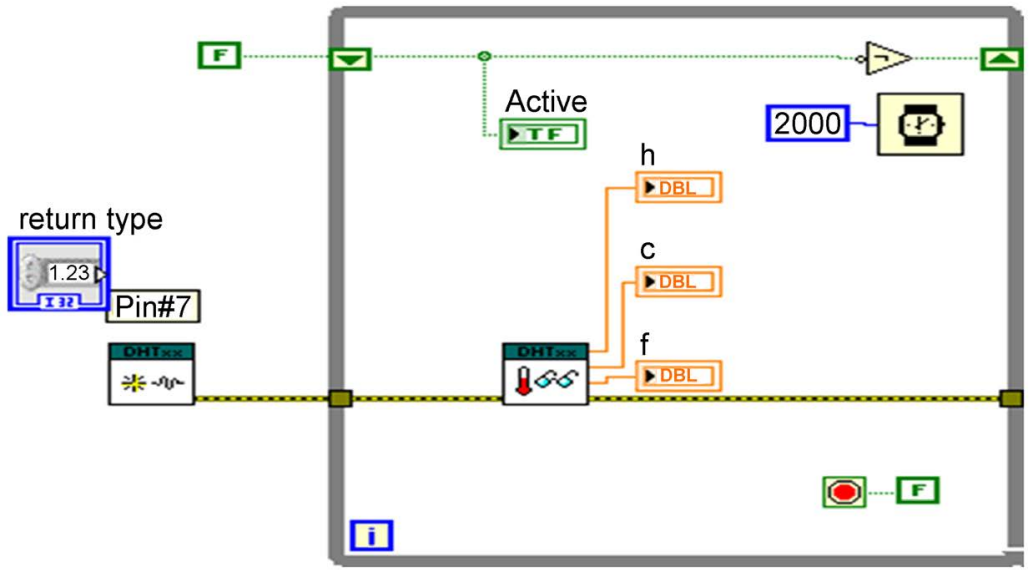

Figure 9. Program for temperature and humidity.

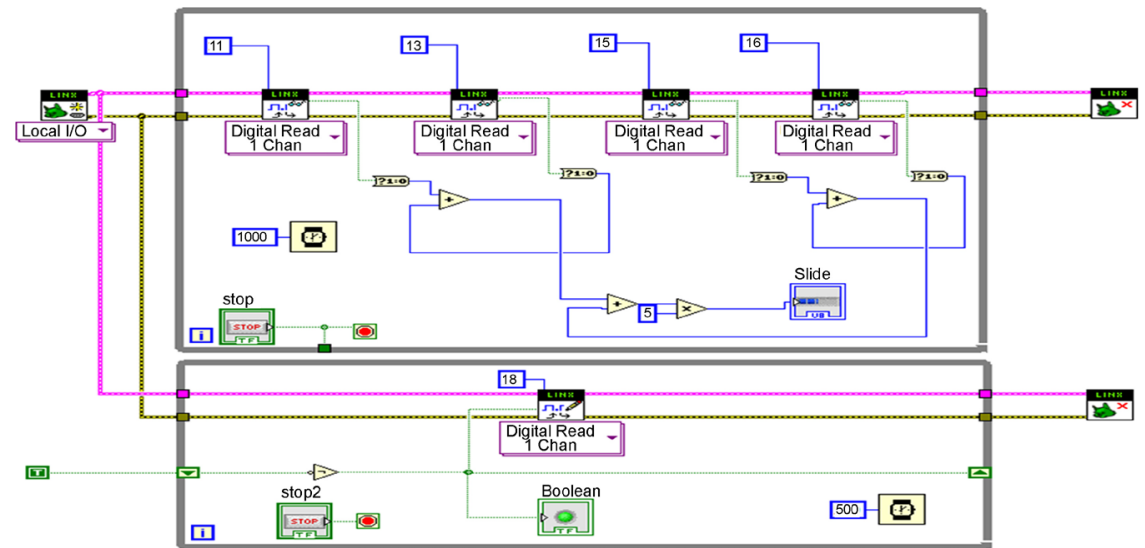

Figure 10. Program for light measurement.

\section{LabVIEW Programming}

The block diagram of the system has been configured by using LabVIEW tools which illustrated in Figure 9 and Figure 10, also the other LabVIEW screen (Virtual Instrument) used to indicate the system results (humidity, temperature, and light intensity) shown in Figure 11 and Figure 12. 


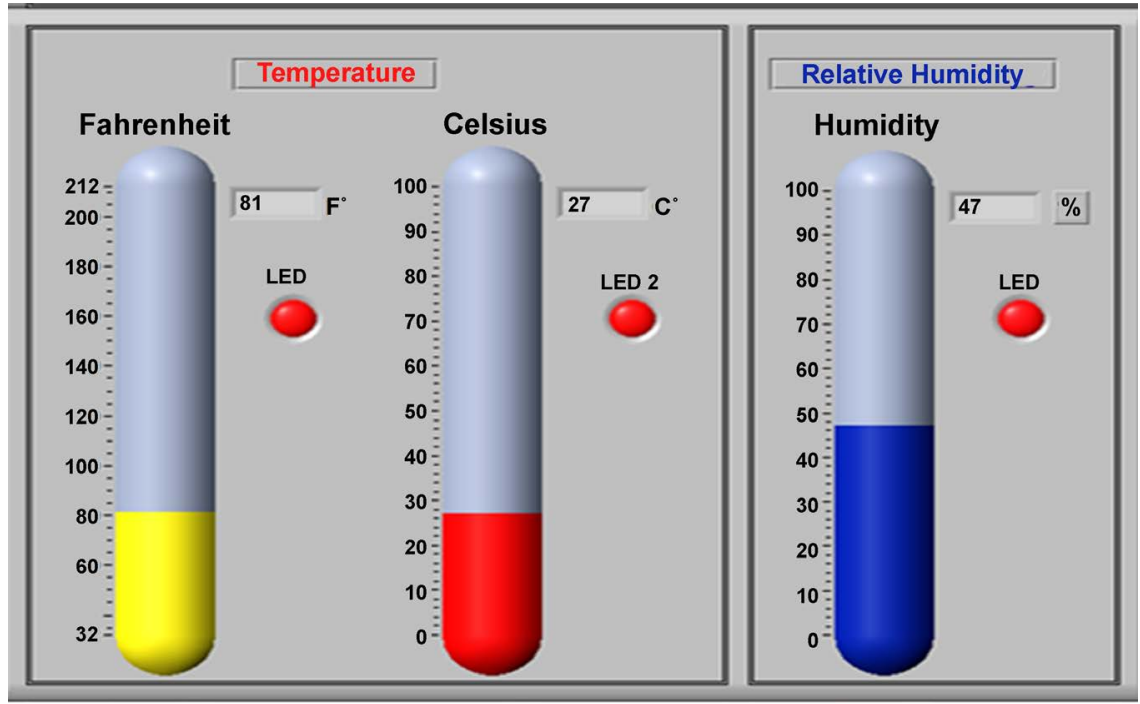

Figure 11. Virtual instrument for illustrated temperature and humidity results.

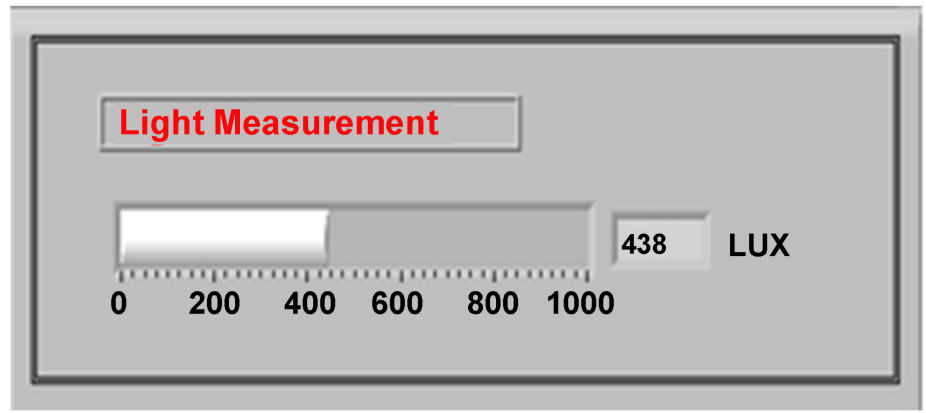

Figure 12. Virtual instrument for illustrated light results.

\section{Conclusion}

This paper has developed real-time monitoring data acquisition from greenhouse via a warless network based on raspberry Pi3. The embedded system implementation improves the growing plant environmental parameter (temperature, humidity, and lighting) in order to improve its productivity. LabVIEW is a powerful platform to design any type of graphical interfacing between the computer and a lot of embedded systems.

\section{Conflicts of Interest}

The authors declare no conflicts of interest regarding the publication of this paper.

\section{References}

[1] Willmott, P.K. (1982) Scientific Greenhouse Gardening. EP Publishing Limited.

[2] Benton Jones, Jr., J. (2007) Tomato Plant Culture in the Field Greenhouse and Home Garden. CRC Press, Boca Raton, FL. https://doi.org/10.1201/9781420007398

[3] Dennis, A.K. (2016) Raspberry Pi Computer Architecture Essentials. Packt Publishing Ltd., UK. 
[4] Yuan, S., Li, W. and Wang, W.W. (2007) Application of Lab VIEW to the Remote Monitoring System for Orchard Environment. Journal of Agricultural Engineering, 23, 186-188. 